\title{
Star formation in the southern dark cloud DC 287.1+02.4
}

\author{
J. L. Yun and P. R. Figueira
}

\begin{abstract}
Centro de Astronomia e Astrofísica da Universidade de Lisboa, Observatório Astronómico de Lisboa, Tapada da Ajuda, 1349-018 Lisboa, Portugal e-mail: [yun;Pedro.Figueira]@oal.ul.pt
\end{abstract}

Received 6 September 2005 / Accepted 23 February 2006

\section{ABSTRACT}

\begin{abstract}
Aims. We report the discovery of a group of 12 new infrared sources seen toward IRAS 10501-5630 and the southern dark globule DC 287.1+02.4. The globule appears as a round patch of extinction in optical images with a typical diameter of 5 arcmin. Methods. The sources are seen on $K \mathrm{~s}$ and $L^{\prime}$ band images taken using SOFI at the NTT and ISAAC at the VLT. The globule was mapped in millimeter molecular transitions ( $\left.\mathrm{CO}(1-0), \mathrm{C}^{18} \mathrm{O}(1-0), \mathrm{C}^{18} \mathrm{O}(2-1), \mathrm{CS}(2-1), \mathrm{HCN}(1-0)\right)$ using the SEST telescope. Results. Millimeter-wave spectroscopy revealed a single dense core seen in $\mathrm{C}^{18} \mathrm{O}, \mathrm{CS}$, and $\mathrm{HCN}$, extending about 2 arcmin. The infrared sources are likely to be embedded in the dense cloud core. The reddest of the new infrared sources, named here DC $287.1+02.4$ IRS, is not detected shortward of $2 \mu \mathrm{m}$, and it exhibits a very red $\left(K \mathrm{~s}-L^{\prime}\right)$ colour. The location and colour of this source suggest that this is the near-infrared counterpart of IRAS 10501-5630. Red nebular emission with an elongated shape is also seen in the $H$ and $K$ s band images and could be due to scattered light originating in the embedded objects.
\end{abstract}

Key words. stars: formation - ISM: clouds - ISM: individual: objects: DC 287.1+02.4 - infrared: ISM - radio lines: ISM ISM: dust, extinction

\section{Introduction}

Most stars seem to form in clusters (Strom et al. 1993) within molecular clouds, either giant molecular clouds or smaller dark clouds. Among the latter, Bok globules have been optically selected as patches of obscuration seen against the background stars. As a result, catalogs of Bok globules (Clemens \& Barvainis 1988; Bourke et al. 1995) tend to include mostly nearby and regular-shaped clouds. These clouds, the smallest and possibly simplest molecular clouds, are thought to produce stars in a single episode of star formation (Yun \& Clemens 1990). Nearby globules are physically small and have been shown to produce just a few stars or small groups of stars. More distant globules may harbour clusters of stars (Yun \& Clemens 1994; Santos et al. 2000).

The dark cloud DC $287.1+02.4$ is listed by Hartley et al. (1986) as a nearly circular cloud $\left(6^{\prime} \times 5^{\prime}\right)$. Despite its typical appearance of a regular-shaped patch of obscuration, it was not included in the Bourke et al. (1995) catalog of southern Bok globules. An IRAS PSC source (IRAS 10501-5630) appears within its boundaries. Pollanen \& Feldman (1995) included this source in their catalog of candidate protostars obtained from the literature, based on the survey by Persi et al. (1990) of young stellar objects (YSOs) in southern dark clouds. In their CS(2-1) survey toward IRAS point sources in the galactic plane, Bronfman et al. (1996) detected CS emission toward IRAS 10501-5630. Henning \& Launhardt (1998) surveyed southern globules for millimeter continuum emission and found a fairly strong point source. Recently Bourke et al. (2005) have surveyed this source for the presence of methanol masers and found none. Henning \& Launhardt (1998) assigned a distance to DC $287.1+02.4$ of $3.1 \mathrm{kpc}$ based on association to the Carina arm.

As part of our study of IRAS sources seen towards molecular material, we have discovered the near-infrared counterpart of IRAS 10501-5630. This source is detected in the $K \mathrm{~s}$ and $L^{\prime}$ bands with no detection shortward of $2 \mu \mathrm{m}$. It exhibits a very red $\left(K s-L^{\prime}\right)$ colour compatible with the presence of excess emission from circumstellar material.

The source is located near the centre of a single dense core in DC $287.1+02.4$ as traced by $\mathrm{CO}, \mathrm{CS}$, and $\mathrm{HCN}$ line transitions. Additional, new infrared sources were also detected in $L^{\prime}$ band images. Together they seem to represent a small cluster of YSOs forming in DC 287.1+02.4.

In this article, we report the discovery of this cluster (including the near-infrared counterpart of IRAS 10501-5630), and characterise its nature and molecular environment.

\section{Observations and data reduction}

\subsection{Infrared imaging}

Broadband $L$ infrared images of IRAS 10501-5630 were obtained on January 13, 2002, in service mode, with the longwavelength arm (Aladdin) of the ISAAC instrument mounted on Unit 1 (Antu) of the ESO Very Large Telescope (VLT). The ISAAC camera (Moorwood et al. 1998) contains a $1024 \times$ 1024 pixel and was used with the pixel scale of $0.07^{\prime \prime}$ per pixel resulting in a field of view of $1.2 \times 1.2 \mathrm{arcmin}^{2}$ on the sky. The $L$ band filter used at ESO on the ISAAC detector is effectively $L^{\prime}$ (3.78 $\mu \mathrm{m}$ with $15 \%$ width).

The observations were carried in chopping and nodding mode, using the ISAACLW_img_obs_AutoChopNod observation template of the Aladdin arm of the ISAAC instrument (Cuby et al. 2005). The chopping throw was $30^{\prime \prime}$ in declination at a frequency of $0.43 \mathrm{~Hz}$. The integration time per frame was $0.11 \mathrm{~s}$. Each on and off image consisted of nine co-added frames. Each exposure included 15 nod cycles following a standard ABBA pattern with a random jitter between each cycle. 
Twenty-four such exposures were taken, and the total integration time was $12 \mathrm{~min}$.

Near-infrared ( $H$ and $K \mathrm{~s}$ ) observations were conducted during December 17, 2002, using the ESO New Technology Telescope (NTT) equipped with SOFI. The SOFI camera contains a $1024 \times 1024$ pixel near-infrared array and was used at a plate scale of $0.29 \mathrm{arcsec} / \mathrm{pixel}$ resulting in a field of view of $4.9 \times 4.9 \operatorname{arcmin}^{2}$ on the sky. At each of 9 different jitter positions (with a jitter box of 30 arcsec), series of 10 images with $2 \mathrm{~s}$ exposure time were taken. The total net integration time is therefore $3 \mathrm{~min}$ in the $H$ and $K$ s filters.

Because $J$-band images were not obtained with the NTT, we used alternative $J$-band images from the $1 \mathrm{~m} \mathrm{YALO}^{1}$ telescope at the Cerro Tololo Inter-American Observatory in Chile equipped with the ANDICAM camera. The ANDICAM near-infrared array contains $1024 \times 1024$ pixels, and it was used at a plate scale of $0.21 \mathrm{arcsec} / \mathrm{pixel}$, resulting in a field of view of $3.6 \times$ $3.6 \operatorname{arcmin}^{2}$ on the sky. We obtained two sets of 7 dithered frames (of 30 arcsec step size and following a pre-defined star-like pattern of the ANDICAM camera). The total integration time per dithered frame was $90 \mathrm{~s}$ at each of the filter bands.

Standard procedures for near-infrared image reduction (JHKs bands) were applied (e.g. Yun \& Clemens 1994) resulting in a final mosaic image for each band. For $L^{\prime}$-band images, on and off images were subtracted. These sky-subtracted images were then shifted and co-added. The resulting image contained negative sources from the subtraction of sources within the chopping distance. However, a careful check of the final images revealed that none of the stars seen had fallen into negative sources.

Source extraction and aperture photometry and correction were performed on each image using IRAF packages following the procedure described in Yun \& Clemens (1995). To avoid contamination from nearby stars, fluxes were obtained in a small aperture $(2 \operatorname{arcsec}$ in $J, 1.7 \operatorname{arcsec}$ in $H$ and in $K \mathrm{~s}$, and $0.85 \operatorname{arcsec}$ in $L^{\prime}$ ) and corrected for the fact that they might not include all the flux from a star. The correction was based on computing, for the brightest stars in each frame, the mean and the dispersion of the differences between the magnitude obtained in the small aperture and the magnitude obtained in a large (virtually infinite) aperture that contained at least $99 \%$ of the bright star fluxes. Finally, experiments with different aperture sizes (small and large) were performed. We concluded that changing the aperture sizes did not significantly alter the photometric results and uncertainties.

Transformation to the standard system was performed using the following standard stars: sj9137, HD 40335, HR 4620, (observed in service mode), from the list of Leggett et al. (2003) and van der Bliek (1996). We estimate the completeness limit of our images at magnitudes $J=15.5, H=18.0, K \mathrm{~s}=18.0, L^{\prime}=13.0$. Photometry errors range from about 0.04 mag for the bright stars to 0.14 mag for the fainter ones.

Astrometry of the final images was performed using GSC and 2MASS stars. For each filter, we looked for stars that could be identified both on GSC (or 2MASS) images and in the central regions of our images. The coordinates of these stars were used to determine the coefficients of the linear transformations that converted pixel coordinates of our images into equatorial coordinates.

${ }^{1}$ YALO is the Yale-AURA-Lisbon-Ohio consortium (Bailyn et al. 1999).

\subsection{Millimetre spectroscopy}

Millimetre observations were carried out using the SwedishESO Submillimetre Telescope (SEST) in Chile during December 15-19, 2002. Spectra were obtained towards IRAS 10501-5630 in the following transitions: $\mathrm{CO}(1-0)$, $\mathrm{C}^{18} \mathrm{O}(1-0), \mathrm{C}^{18} \mathrm{O}(2-1), \mathrm{CS}(2-1)$, and $\mathrm{HCN}(1-0)$. For the different $\mathrm{CO}$ transitions and for $\mathrm{CS}$, several positions were observed in an approximately full-beam spacing grid, in order to produce small maps $(5 \times 5$ for $\mathrm{CO} ; 3 \times 3$ for $\mathrm{CS})$. The pointing was checked regularly and the error found to be better than 7 arcsec. We used SIS receivers and the 2000-channel acousto-optical spectrometer as a back-end, with a resolution of $43 \mathrm{kHz}$ per channel $\left(0.11 \mathrm{~km} \mathrm{~s}^{-1}\right.$ at $\left.115 \mathrm{GHz}\right)$. As observing modes, frequency switching and double-beam switching modes were used.

Spectral line intensities were calibrated and corrected for atmospheric losses using the standard chopper wheel method to obtain the antenna temperature $T_{\mathrm{A}}^{*}$. To get main-beam radiation temperatures $T_{\mathrm{R}}, T_{\mathrm{A}}^{*}$ was divided by the main-beam efficiency $\eta_{\mathrm{mb}}\left(\eta_{\mathrm{mb}}=0.75\right.$ at $86 \mathrm{GHz}, \eta_{\mathrm{mb}}=0.70$ at $115 \mathrm{GHz}$, $\eta_{\mathrm{mb}}=0.60$ at $\left.230 \mathrm{GHz}\right)$.

The data were processed using standard procedures of the Continuum and Line Analysis Single-dish Software (CLASS) package developed at the Observatoire de Grenoble and IRAM Institute. Baselines were fitted and removed, followed by Gaussian fitting, yielding the values for the line parameters (peak, width, and integrated intensity). Typical $1 \sigma$ rms values were $0.17 \mathrm{~K}, 0.09 \mathrm{~K}, 0.09 \mathrm{~K}, 0.08 \mathrm{~K}$, and $0.05 \mathrm{~K}$, for $\mathrm{CO}(1-0)$, $\mathrm{C}^{18} \mathrm{O}(1-0), \mathrm{C}^{18} \mathrm{O}(2-1), \mathrm{CS}(2-1)$, and $\mathrm{HCN}(1-0)$, respectively.

\section{Results and discussion}

\subsection{Optical extinction, CO map, and dense core properties}

In Fig. 1, we present the SEST CO $(J=1-0)$ map of velocityintegrated intensity towards IRAS 10501-5630 superimposed on the optical image from the Digitized Sky Survey. The patch of obscuration clearly indicates the location of the cloud. The CO line contours exhibit almost circular shapes becoming elongated towards the east. The western edge of the cloud is sharp, whereas the gradient of the $\mathrm{CO}$ intensity at the eastern edge is shallower. Our CO map, with a size of approximately $3.8^{\prime} \times 3.8^{\prime}$, does not cover the whole extent of the cloud. The $\mathrm{CO}$ emission is very strong with the first contour at $18 \mathrm{~K} \mathrm{~km} \mathrm{~s}^{-1}$. The strongest emission is seen close to the position of the IRAS PSC object located at the centre of the image.

Analysis of the CO and CS spectra, obtained in beamswitching mode, revealed that the lines appear regular and close to Gaussian, at $v_{\mathrm{LSR}}=-13.0 \mathrm{~km} \mathrm{~s}^{-1}$. No significant asymmetry, extended wings, or multiple components have been detected. Table 1 gives the millimeter line parameters at the position of IRAS 10501-5630, which is also the position of maximum emission for all transitions measured. The CS line is relatively strong (e.g. Launhardt et al. 1998), in agreement with the presence of a dense molecular core.

In Fig. 2, we present the $\mathrm{C}^{18} \mathrm{O}(2-1)$ and $\mathrm{CS}(2-1)$ maps. At the available resolution, only one dense core is detected, with a regular, round shape and centred close to IRAS 10501-5630. The $\mathrm{C}^{18} \mathrm{O}(2-1)$ map extends about $80 \operatorname{arcsec}(1.2 \mathrm{pc})$ (to the halfintensity contour). The $\mathrm{CS}(2-1)$ map extends about 72 arcsec (1.2 pc).

We estimated the mass of the dense core using $\mathrm{C}^{18} \mathrm{O}$ data. Excitation temperatures were determined for each beam where 


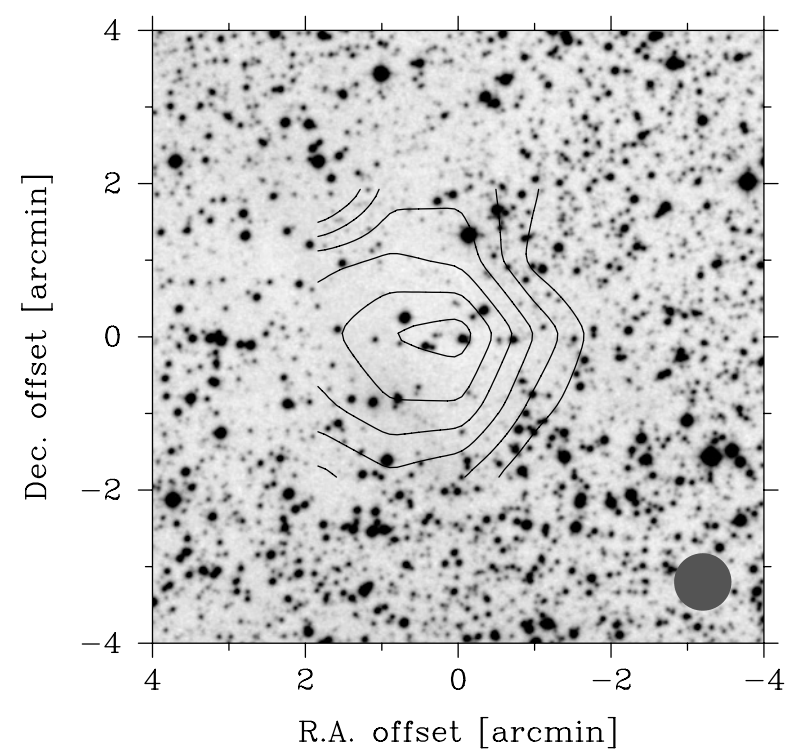

Fig. 1. Optical image of the region towards IRAS 10501-5630 from the Digitized Sky Survey. Superimposed on the image is the velocityintegrated SEST CO $(J=1-0)$ intensity map (contour lines). The $\mathrm{CO}$ emission is very strong with the first contour at $18 \mathrm{~K} \mathrm{~km} \mathrm{~s}^{-1}$. Subsequent contours are at steps of $7 \mathrm{~K} \mathrm{~km} \mathrm{~s}^{-1}$. The strongest emission is seen close to the position of the IRAS PSC object located at the centre of the image.

Table 1. Millimeter line parameters of DC $287.1+02.4$ at the position of IRAS 10501-5630.

\begin{tabular}{lccc}
\hline \hline & $\begin{array}{c}T_{\mathrm{R}} \\
(\mathrm{K})\end{array}$ & $\begin{array}{c}\int T_{\mathrm{R}} \mathrm{d} v \\
\left(\mathrm{~K} \mathrm{~km} \mathrm{~s}^{-1}\right)\end{array}$ & $\begin{array}{c}\Delta v_{\mathrm{FWHM}} \\
\left(\mathrm{km} \mathrm{s}^{-1}\right)\end{array}$ \\
\hline $\mathrm{CO}(1-0)$ & $16.27(0.17)$ & $54.12(0.15)$ & $3.20(0.01)$ \\
$\mathrm{C}^{18} \mathrm{O}(1-0)$ & $1.28(0.09)$ & $1.88(0.06)$ & $1.38(0.05)$ \\
$\mathrm{C}^{18} \mathrm{O}(2-1)$ & $1.71(0.09)$ & $2.51(0.04)$ & $1.38(0.03)$ \\
$\mathrm{CS}(2-1)$ & $1.25(0.08)$ & $2.26(0.06)$ & $1.69(0.05)$ \\
$\mathrm{HCN}^{a}(1-0)$ & $0.90(0.05)$ & $2.96(0.07)$ & $1.98(0.13)$ \\
\hline
\end{tabular}

${ }^{a}$ The main component of the three hyperfine transitions.

both $\mathrm{C}^{18} \mathrm{O}(2-1)$ and $\mathrm{C}^{18} \mathrm{O}(1-0)$ were detected. The temperatures were found to be within a narrow range, from 5 to $9 \mathrm{~K}$. The LTE conditions were then assumed for each beam in order to estimate total column densities, yielding the value of $M \sim 400\left(\frac{D}{3100 \mathrm{pc}}\right)^{2} M_{\odot}$ for the mass of the cloud core. A similar approximation leads to a rough estimate of the volume density at the central position, $n\left(\mathrm{H}_{2}\right)$, of about $3 \times 10^{4} \mathrm{~cm}^{-3}$.

Using the CO map and standard conversions of integrated $\mathrm{CO}$ intensity to $\mathrm{H}_{2}$ column density (e.g. Goldsmith 1999), we estimate a lower limit for the mass of the cloud core of $230\left(\frac{D}{3100 \mathrm{pc}}\right)^{2} M_{\odot}$, in good agreement with the estimate based on the $\mathrm{C}^{18} \mathrm{O}$ data. These values compare well with a virial mass of about $310\left(\frac{D}{3100 \mathrm{pc}}\right) M_{\odot}$ for the cloud core indicating that the core is virialized and bound.

Using standard $N\left(\mathrm{H}_{2}\right)$ to $A_{\mathrm{v}}$ conversion factors (Bohlin et al. 1978), we estimate an optical extinction of $A_{\mathrm{v}} \sim 10 \mathrm{mag}$ through the centre of the cloud core. This is compatible with the value of $A_{\mathrm{v}} \sim 18$ derived from the dust emission $1.3 \mathrm{~mm}$ flux.
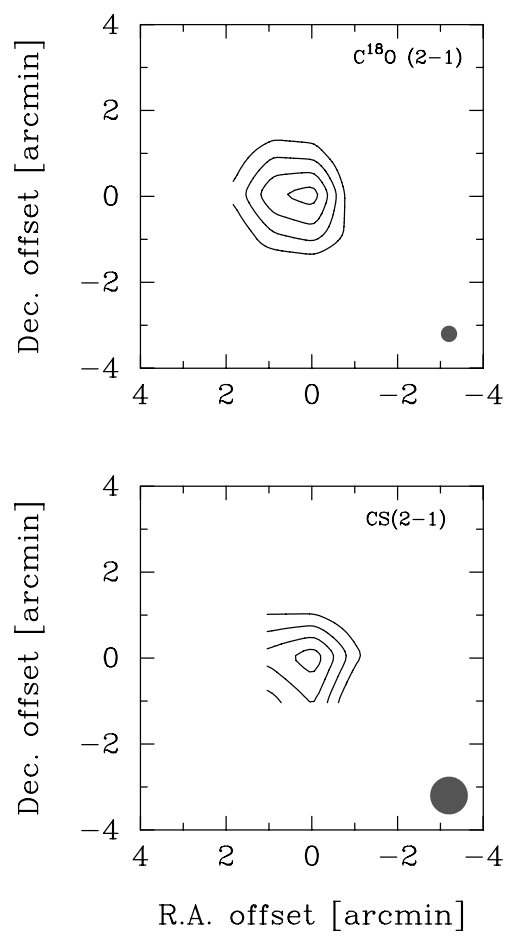

Fig. 2. Maps of $\mathrm{C}^{18} \mathrm{O}(2-1)$ integrated intensity and of $\mathrm{CS}(2-1)$ peak radiation temperature. The maps are centred at the IRAS position. The first contour in the $\mathrm{C}^{18} \mathrm{O}(2-1)$ map is at $0.80 \mathrm{~K} \mathrm{~km} \mathrm{~s}^{-1}$. Subsequent contours are at steps of $0.45 \mathrm{~K} \mathrm{~km} \mathrm{~s}^{-1}$. The first contour in the $\mathrm{CS}(2-1)$ map is at $0.50 \mathrm{~K}$. Subsequent contours are at steps of $0.20 \mathrm{~K}$.

\subsection{The near-infrared images towards IRAS 10501-5630: a group of embedded objects}

In Fig. 3, we show the central regions of the $H$-band, the $K$ s-band, and the $L^{\prime}$-band final infrared-images of IRAS 105015630. The images cover about $1.1 \times 1.1 \mathrm{arcmin}^{2}$. Notice the presence of nebular emission in the $K$ s-band image around the central region.

\subsubsection{Nebular emission}

The ellipse at the centre of Fig. $3 \mathrm{~b}$ represents the IRAS PSC error ellipse. Even at $2.2 \mu \mathrm{m}$, a dense opaque region can be seen near the centre of the image. Around the IRAS position, several objects appear together with some nebular emission.

Several stars near the centre of the $H$ and $K$ s band images are surrounded by nebular emission. An elongated structure is also seen. This nebulosity suggests that some of these stars are associated to the cloud, being either embedded or just emerging from the cloud. Given the absence of any nebular emission in the optical DSS image, the nebulosity seen in the $H$ and $K$ s band images could be due to light from young embedded stars escaping from the interior of the cloud core through holes or cavities excavated by stellar winds.

In order to distinguish which of the stars appearing on the images are likely to be embedded objects and possibly responsible for the nebular emission seen, we analysed their infrared colours.

\subsubsection{Infrared colours}

On the $J$ band image, only a few and only the relatively bright and blue sources could be detected. Their $(J-H)$ and 

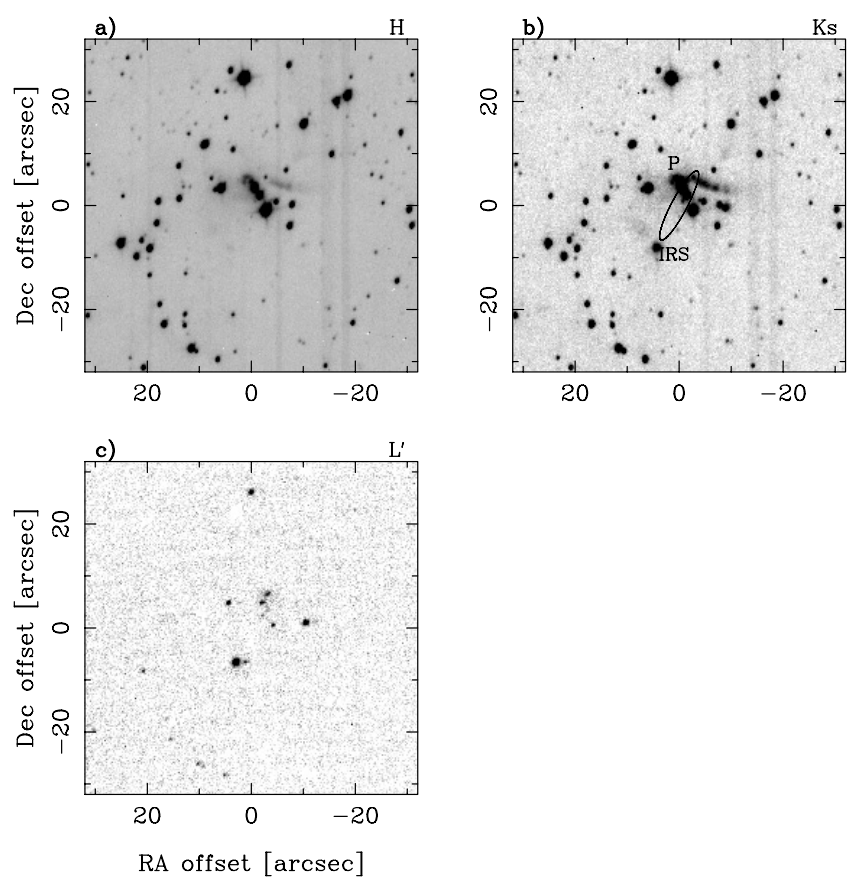

Fig. 3. Central regions of the $H$-band, the $K$ s-band, and the $L^{\prime}$-band infrared-images of IRAS 10501-5630. The images cover about $1.1 \times$ $1.1 \mathrm{arcmin}^{2}$. North is up and east to the left. Several stars near the centre of the $H$ and $K \mathrm{~s}$ band images are surrounded by nebular emission. The ellipse in the $K$ s-band image is the IRAS PSC error ellipse. Notice also the source located to the southeast (offsets: $+4,-7$ ) along the major axis of the error ellipse, labeled "IRS". The letter "P" represents the location of the near-infrared source found by Persi et al. (1990).

( $H-K \mathrm{~s})$ colours indicate that, with two exceptions, all the sources detected simultaneously in $J, H$, and $K$ s bands are likely to be foreground and background objects not embedded in the cloud core. The two exceptions are listed in Table 2 (see text below).

On the other hand, the $L^{\prime}$-band image contains much richer information. Eighteen sources were detected in this band. Out of these 18 sources, 6 sources are relatively blue and appear scattered throughout the image. These bluer sources also appear in the $J, H$, and $K$ s images and their $(J-H)$ and $(H-K \mathrm{~s})$ colours indicate that they are not embedded in the cloud core. Their colours are consistent with those of main-sequence or giant stars reddened by dust following a normal interstellar extinction law (Rieke \& Lebofsky 1985).

Of the remaining 12 sources, 9 have values $\left(K \mathrm{~s}-L^{\prime}\right) \geq 1.5$, and 3 sources have been detected only in the $L^{\prime}$ band image ( $L^{\prime}$ sources). These 12 sources are likely to be embedded sources (e.g. Lada et al. 2004). In fact, they exhibit colour excesses consistent with those of YSOs surrounded by circumstellar material. Six of these sources have simultaneous $H, K$ s, and $L^{\prime}$ band photometries, allowing their positions to be checked in the $\left(K s-L^{\prime}\right)$, $(H-K \mathrm{~s})$ colour-colour diagram. This is presented in Fig. 4. All six sources are located to the right of the reddening band suggesting the presence of infrared excess emission typical of YSOs surrounded by circumstellar material.

These facts suggest that the red sources detected in the $L^{\prime}$ band image represent a small cluster of YSOs embedded in a dense core contained in the dark globule DC 287.1+02.4. In Table 2, we give the photometry of these sources (uncertainties are about $0.10 \mathrm{mag}$ ). The astrometry was obtained using GSC and 2MASS stars.
Table 2. Photometry of red sources seen on the $L^{\prime}$ band image towards DC 287.1+02.4.

\begin{tabular}{lcccccc}
\hline \hline ID & $\begin{array}{c}\text { RA } \\
(2000)\end{array}$ & $\begin{array}{c}\text { Dec } \\
(2000)\end{array}$ & $m_{J}$ & $m_{H}$ & $m_{K}$ & $m_{L}$ \\
& 105213.8 & -564635 & $\ldots$ & $\ldots$ & 15.5 & 10.5 \\
\hline 3 & 105214.7 & -564630 & $\ldots$ & $\ldots$ & $\ldots$ & 12.1 \\
$5^{a}$ & 1052534.8 & -564634 & $\ldots$ & $\ldots$ & $\ldots$ & 13.1 \\
6 & 105214.8 & & & \\
7 & 105214.8 & -564631 & $\ldots$ & 14.9 & 13.7 & 11.6 \\
9 & 105215.2 & -564643 & $\ldots$ & $\ldots$ & $\ldots$ & 12.1 \\
$10^{b}$ & 105215.5 & -564643 & $\ldots$ & $\ldots$ & 15.3 & 9.5 \\
11 & 105215.6 & -564631 & 15.4 & 13.9 & 13.0 & 11.2 \\
12 & 105215.7 & -564705 & $\ldots$ & 16.3 & 14.6 & 12.4 \\
13 & 105216.3 & -564704 & $\ldots$ & $\ldots$ & 15.3 & 13.1 \\
15 & 105217.0 & -564658 & 16.4 & 15.1 & 14.2 & 12.6 \\
16 & 105217.7 & -564645 & $\ldots$ & 15.5 & 14.1 & 12.1 \\
18 & 105218.9 & -564657 & $\ldots$ & 16.8 & 15.3 & 12.6 \\
\hline
\end{tabular}

${ }^{a}$ Nebular. ${ }^{b}$ Nebular, IRS.

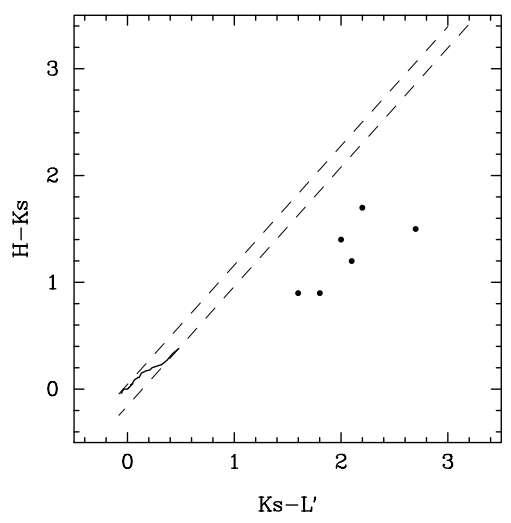

Fig. 4. $\left(K S-L^{\prime}\right),(H-K s)$ colour-colour diagram for sources that were detected simultaneously in $H, K \mathrm{~s}$, and $L^{\prime}$ bands. Their location to the right of the reddening band indicates the presence of infrared excess emission.

Source No. 5 (in Table 2) is marked "Nebular". It was detected only on the $L^{\prime}$ band image and has a nebulous appearance with a full-width at half-maximum (FWHM) that is much larger than those of other point sources. Its spatial position is well aligned with the elongated structure seen in the $H$ and $K \mathrm{~s}$ band images and so they could be related. Additional data (e.g. $\mathrm{H}_{2}$ narrow-band images) would be needed for further conclusions.

The six sources in Table 2 with simultaneous $H, K \mathrm{~s}$, and $L^{\prime}$ photometry have $(H-K \mathrm{~s}),\left(K \mathrm{~s}-L^{\prime}\right)$ colours that, in the corresponding colour-colour diagram, place them well outside the reddening band, thus indicating the presence of infrared excess emission above that of a stellar photosphere.

\subsection{The near-infrared counterpart of IRAS 10501-5630}

In Fig. 3b, notice in particular the source labeled "IRS", located just to the southeast along the major axis of the error ellipse (close to the southern tip of the ellipse) at offsets $(\Delta \alpha, \Delta \delta)=$ $(+4,-7)$. This source, which we name DC $287.1+02.4$ IRS, exhibits very red colours, as can be verified in a careful inspection of Fig. 3. It is not detected in the $H$ band image. The source appears point-like but is surrounded by faint nebular emission. A PSF fit reveals a full-width at half-maximum (FWHM) of 1.0 arcsec, larger than that of the other real point-sources across 
the image $(0.6$ arcsec). As can be verified by examining Fig. 3 and Table 2, DC 287.1+02.4 IRS is the brightest source in the $L^{\prime}$ band image and it has the reddest $\left(K \mathrm{~s}-L^{\prime}\right)$ colour.

We take these facts, together with the position very close to the IRAS PSC error ellipse and along its major axis, as fairly good evidence that DC 287.1+02.4 IRS is likely to be the nearIR counterpart of IRAS 10501-5630 (however, see below).

The position of DC 287.1+02.4 IRS on the sky is offset from IRAS $10501-5630$ by +4 arcsec in right ascension and -9 arcsec in declination. These offsets do not coincide with those of the near-infrared source found by Persi et al. (1990) using an InSb infrared photometer in a raster scan mode. Their source had offsets of $(+1,+7)$ and is located slightly outside the error ellipse to the north of IRAS 10501-5630. Given the large aperture (15") of the diaphragm they used, their source is likely to be composed of several sources present in our Ks-band image.

Like the other sources listed in Table 2, DC 287.1+02.4 IRS is likely to be embedded in the cloud and thus be a young stellar object. In fact, if it were a background source and its red colour were due to extinction through the cloud core, we could use the value of the $\left(K \mathrm{~s}-L^{\prime}\right)$ colour index to derive a lower limit to the extinction $A_{\mathrm{V}}$ through the centre of the core. Using the interstellar extinction law of Rieke \& Lebofsky (1985), we obtain $A_{\mathrm{V}}=18.5 E_{K-L}$. In order to obtain a lower limit for $A_{\mathrm{V}}$, we assume that DC $287.1+02.4$ IRS is a red main-sequence star or a supergiant star with intrinsic $\left(K s-L^{\prime}\right)$ of abou 0.4 (Bessell \& Brett 1988). This leads to $A_{\mathrm{V}} \geq 100 \mathrm{mag}$, a very large value and unlikely for a regular cloud core. Extinctions at this order of magnitude are more typical of circumstellar material either in the form of an envelope or of a disc or both. Thus, we conclude that DC 287.1+02.4 IRS should be a young stellar object embedded within the cloud core.

Figure 5 presents the infrared and millimeter spectral energy distribution (SED) of IRS, assuming it is the near-infrared counterpart of IRAS 10501-5630. The $L^{\prime}$ band flux seems to be somewhat higher than expected for a non-detection by IRAS at $12 \mu \mathrm{m}$. This could be due to uncertainties in the calibration of the $L^{\prime}$ band flux or be due to source variability. This could also indicate that IRS might not be the near-infrared counterpart of IRAS 10501-5630. In this case, the IRAS detection would have been triggered by a redder, fainter source, which was not seen in our images. In any case, this would not affect our conclusions about the YSO-nature of IRS.

The slopes of the SED are consistent with the SED of a young embedded object. Again assuming IRS is the near-IR counterpart of IRAS 10501-5630, we computed the values of the spectral indices i.e., $\alpha=\mathrm{d} \log \left(\lambda S_{\lambda}\right) / \mathrm{d} \log \lambda$ (where $\lambda$ is the wavelength and $S_{\lambda}$ the flux density) between 2.2 and $12 \mu \mathrm{m}$ and between 2.2 and $25 \mu \mathrm{m}$. Because the $12 \mu \mathrm{m}$ flux is an upper limit, we obtain $\alpha(2.2 / 12) \leq 2.67$ and $\alpha(2.2 / 25)=2.19$. In both cases, the large positive values of $\alpha$ support the conclusion that IRS is a YSO in an early stage of evolution.

From the $K \mathrm{~s}$ and $L^{\prime}$-band photometry, the IRAS fluxes, and the $1.3 \mathrm{~mm}$ flux given by Henning \& Launhardt (1998), the luminosity of DC $287.1+02.4$ IRS can be estimated to be $500\left(\frac{D}{3100 \mathrm{pc}}\right)^{2} L / L_{\odot}$. About $65 \%$ of this luminosity comes from the $100 \mu \mathrm{m}$ IRAS flux, which is likely to include a significant contribution of dust emission from the cloud. Also due to the very large IRAS beam at this wavelength, it is likely to include contamination from the other sources present in the image. Given the large uncertainty in the distance assigned to the cloud, no other reliable conclusions should be extracted from this estimate of the luminosity.

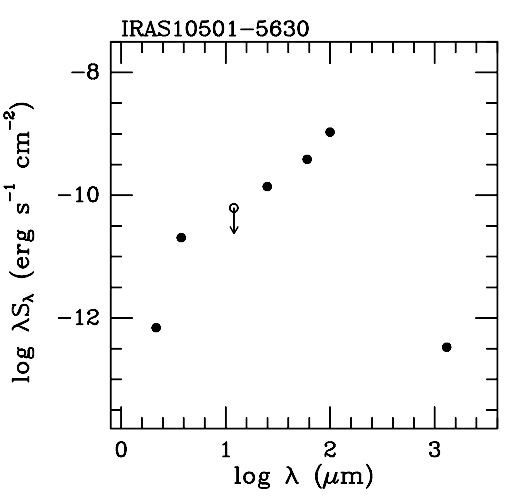

Fig. 5. Spectral energy distribution of IRS. The data points correspond to $1.3 \mathrm{~mm}$, IRAS, $L^{\prime}$, and $K \mathrm{~s}$.

\section{Conclusions}

1. We have discovered a group of 12 YSOs seen on the $L^{\prime}$ band image towards IRAS 10501-5630. Among these sources, we identified the source DC $287.1+02.4$ IRS, the reddest and brightest source detected in our infrared images. This source is likely to be the near-infrared counterpart of IRAS 10501-5630. It is located close to the tip of the IRAS PSC error ellipse, with offsets of $4 \operatorname{arcsec}$ east and $9 \operatorname{arcsec}$ south of IRAS 10501-5630. The source is detected in the $K$ s and $L^{\prime}$ bands with no detection shortward of $2 \mu \mathrm{m}$. It exhibits a very red $\left(K s-L^{\prime}\right)$ colour that is compatible with the presence of excess emission, above that of a stellar photosphere, due to circumstellar material.

2. This group of YSOs is located near the centre of a single dense core in DC $287.1+02.4$ as traced by CO, CS, and HCN millimeter line transitions.

Acknowledgements. This work was partly supported by the Portuguese Fundação para a Ciência e Tecnologia (FCT).

\section{References}

Bessel, M. S., \& Brett, J. M. 1988, PASP, 100, 1134

Bailyn, C., DePoy, D., Agostinho, R., et al. 1999, The First Year of Operations of the YALO Consortium, A\&AS, 195, 8706

Bohlin, R. C., Savage, B. D., \& Drake J. F. 1978, ApJ, 224, 132

Bourke, T. L., Hyland, A. R., \& Robinson, G. 1995, MNRAS, 276, 1052

Bourke, T. L., Hyland, A. R., \& Robinson, G. 2005, ApJ, 625, 883

Bronfman, L., Nyman, L. A., \& May, J. 1996, A\&AS, 115, 81

Clemens, D. P., \& Barvainis, R. E. 1988, ApJS, 68, 257

Cuby, J. G., Lidman, C., Johnson, R., et al. 2005, in Very Large Telescope (ESO on-line publication), 83

Goldsmith, P. F. 1999, in Millimeter-Wave Astronomy: Molecular Chemistry \& Physics in Space, 57, 94

Hartley, M., Tritton, S. B., Manchester, R. N., Smith, R. M., \& Goss, W. M. 1986, A\&AS, 63, 27

Henning, T., \& Launhardt, R. 1998, A\&A, 338, 223

Lada, C. J., Muench, A. A., Lada., E., \& Alves, J. 2004, AJ, 128, 1254

Launhardt, R., Evans, N. J., Wang, Y., et al. 1998, ApJS, 119, 59

Leggett, S. K., Hawarden, T. G., Currie, M. J., et al. 2003, MNRAS, 345, 144

Moorwood, A., Cuby, Y.-G., Biereichel, P., et al. 1998, Messenger, 94, 7

Persi, P., Ferrari-Toniolo, M., Busso, M., et al. 1990, AJ, 99, 303

Pollanen, M. D., \& Feldman, P. A. 1995, PASP, 107, 617

Rieke, G. H., \& Lebofsky, M. J. 1985, ApJ, 288, 618

Santos, C. A., Yun, J. L., Clemens, D. P., \& Agostinho, R. J. 2000, ApJ, 540, L87

Strom, K. M., Strom, S. E., \& Merril, K. M. 1993, ApJ, 412, 233

van der Bliek, N. S., Manfroid, J., \& Bouchet, P. 1996 A\&AS, 119, 547

Yun, J. L., \& Clemens, D. P. 1990, ApJ, 365, L73

Yun, J. L., \& Clemens, D. P. 1994, AJ, 108, 612

Yun, J. L., \& Clemens, D. P. 1995, AJ, 109, 742 